\title{
INFLUÊNCIA DO EQUIPAMENTO NA CINÉTICA DE CRISTALIZAÇÃO DO SORO CONCENTRADO
}

\section{The influence of crystallization equipment on the crystallization of concentrated whey}

\author{
Moisés Simeãol, Rodrigo Stephani ${ }^{2}$, Guilherme Miranda Tavares ${ }^{3}$, \\ Antônio Fernandes Carvalho ${ }^{1}$, Pierre Schuck ${ }^{4}$, Italo Tuler Perrone ${ }^{I^{*}}$
}

\begin{abstract}
RESUMO
O principal objetivo deste trabalho consistiu na determinação do efeito do tipo de cristalizador na eficiência da cristalização da lactose em soro de leite concentrado, utilizando dois equipamentos que dispõem de diferentes formas de agitação (tanque 1 - agitador central e tanque 2 - agitador periférico), mantendo as demais condições de cristalização constantes, como: taxa de resfriamento; temperatura; velocidade de agitação; tempo de cristalização e teor se sólidos solúveis por tratamento. A maior taxa de cristalização no soro concentrado foi obtida pelo emprego da agitação central $[$ Tempo $(\min )=0,1927(\%$ cristalização da lactose $)+21,681]$ e $[$ Tempo $(\min )=$ $-0,06\left({ }^{\circ}\right.$ Brix $\left.)+51,233\right]$ sob a taxa de resfriamento de $-0,34{ }^{\circ} \mathrm{C} \pm 0,05{ }^{\circ} \mathrm{C}$ por minuto, durante a primeira hora e alcançando a temperatura de $25,8^{\circ} \pm 1,2{ }^{\circ} \mathrm{C}$ após 4 horas. A agitação central promoveu as maiores taxas de cristalização em soro concentrado com $50{ }^{\circ} \mathrm{Brix}, 55^{\circ} \mathrm{Brix}$ e $60^{\circ} \mathrm{Brix}$. Observou-se que mudanças nas condições de cristalização do soro concentrado (em diferentes tanques) e no teor de sólidos solúveis implicam na obtenção de pós com diferentes propriedades de reidratação, de adesão e com teores distintos de lactose cristalizada, impactando na conservação durante o prazo de validade.
\end{abstract}

Palavras-chave: tanque; geometria; cisalhamento; agitação; cristalização.

1 Universidade Federal de Viçosa (UFV), Av. Peter Henry Rolfs, s/n, Campus Universitário, 36570-900, Viçosa, MG, Brasil. E-mail: italotulerperrone@gmail.com

2 Universidade Federal de Juiz de Fora (UFJF), Juiz de Fora, MG, Brasil.

3 Universidade Estadual de Campinas (UNICAMP), Campinas, SP, Brasil.

4 UMR STLO, INRA, Agrocampus-Ouest, França.

* Autor para correspondência.

Recebido / Received: 29/06/2016

Aprovado / Approved: 17/05/2017 


\begin{abstract}
The main objectives of this project were to determine the effect of the crystallizer design on lactose crystallization efficiency in concentrated whey. Two different crystallizers were used, one presenting a central agitation (tank 1) and the other presenting a peripheral agitation (tank 2). All others crystallization parameters were kept constant such as cooling rate; temperature; stirring speed; crystallization time and concentration of soluble solids by treatment. The best rate for lactose crystallization in concentrated whey was obtained applying central stirring and $55^{\circ}$ Brix [Time $(\mathrm{min})=0.1927(\%$ lactose crystallization $\left.)+21.681\right]$ and $[$ Time $(\mathrm{min})$ $=-0.06$ ( ${ }^{\circ}$ Brix $\left.)+51.233\right]$ under the cooling rate of $-0.34{ }^{\circ} \mathrm{C} \pm 0.05{ }^{\circ} \mathrm{C}$ per minute during the first hour and achieving the final temperature of $25.8^{\circ} \pm 1.2^{\circ} \mathrm{C}$ after 4 hours, central stirring promoted highest rates of lactose crystallization in concentrated whey with 50, 55 and $60^{\circ}$ Brix. The type of Tank for crystallization and the dry matter content in concentrated whey affect whey powder rehydration, stickiness into the chamber, different levels of lactose crystallization and storage of the powder.
\end{abstract}

Keywords: tank; design; shear; stirring; crystallization.

\section{INTRODUÇÃO}

Entende-se por soro em pó o produto obtido pela desidratação do soro ou soro ácido, mediante processos tecnologicamente adequados, aptos para a alimentação humana. Para a elaboração deste produto as principais operações unitárias envolvidas são: separação por membranas, evaporação a vácuo, cristalização e secagem em spray dryer. Segundo Masters (2002), o soro em pó, quando obtido sem prévia cristalização, é um pó com baixa densidade, higroscópico e com grande tendência a agregação de partículas (caking) e a adesão no spray dryer (stickiness). Desta forma, é etapa obrigatória para a produção do soro em pó, a cristalização da lactose no soro concentrado.

De acordo com Písecký (1997), para uma cristalização eficiente, a fim de evitar a transição vítrea durante a secagem ou estocagem, é necessária a cristalização de no mínimo $70 \%$ da lactose presente no soro concentrado. A cristalização da lactose é bem descrita na literatura científica, contudo existem poucos trabalhos que relacionam o tipo de tanque de cristalização com a taxa de formação dos cristais.

O objetivo deste trabalho foi avaliar o efeito do tipo de cristalizador na eficiência da cristalização da lactose em soro de leite concentrado por meio de dois equipamentos que dispõem de diferentes formas de agitação (Tanque 1 - com agitador central e Tanque 2 - com agitador periférico).

\section{MATERIAL E MÉTODOS}

O delineamento experimental (Figura 1) foi composto de seis tratamentos $(\mathrm{T})$ no qual foram utilizados dois tanques de cristalização: tanque 1 (com agitador central) e tanque 2 (com agitador periférico) e três concentrações de sólidos solúveis ao final da evaporação $\left(50{ }^{\circ} \mathrm{Brix}, 55^{\circ} \mathrm{Brix}\right.$ e $\left.60{ }^{\circ} \mathrm{Brix}\right)$. Empregouse evaporador (tacho) de único efeito da marca $\operatorname{Treu}^{\circledR}(45-55 \mathrm{~cm} \mathrm{Hg})$, alimentado por soro pré-concentrado desmineralizado (33 \pm $1{ }^{\circ}$ Brix) obtido em parceria com a Indústria de Laticínios Porto Alegre, Ponte Nova, MG.

A concentração do soro foi determinada através de um refratômetro digital da marca 
Biobrix ${ }^{\circledR}$ modelo 2WAJ-D. A cristalização do soro foi realizada imediatamente após o término da evaporação a vácuo. $\mathrm{O}$ tanque de cristalização 1 (tacho aberto de camisa dupla da marca Inoxul ${ }^{\circledR}$ com capacidade de $50 \mathrm{~kg}$ provido de um agitador central) e o tanque de cristalização 2 (tanque misturador da marca Global Inox ${ }^{\circledR}$ com capacidade para $100 \mathrm{~kg}$ com agitador periférico) são apresentados na Figura 2.

A rotação foi padronizada em ambos tanques para 50 rotações por minuto, contudo este procedimento não padronizou a velocidade tangencial do fluido durante a cristalização. $\mathrm{O}$ resfriamento foi iniciado juntamente com a adição do soro nos tanques de cristalização por meio da circulação indireta na camisa do equipamento de água industrial a temperatura média de $24 \pm 2{ }^{\circ} \mathrm{C}$, até a temperatura do soro atingir valor próximo a $30^{\circ} \mathrm{C}$. O volume de soro adicionado em cada um dos tanques para a cristalização foi de $40 \%$ em relação a sua capacidade, suficiente para cobrir a superfície do agitador. O tempo de permanência no cristalizador foi de 240 minutos. Para o estabelecimento da cinética de cristalização foram coletadas amostras de soro nos dois tanques em intervalos de 20 minutos (tempo zero até 240 minutos) e em pontos de coleta específicos (Figura 3).

A partir dos resultados obtidos foram determinados os percentuais de cristalização da lactose de acordo com a Equação 1 sugerida por Westergaard (2004).

$$
\% \mathrm{Cr}=\frac{\left(\mathrm{B}_{1}-\mathrm{B}_{2}\right) \times 9500 \times 100}{\mathrm{LAC} \times \mathrm{EST} \times\left(95-\mathrm{B}_{2}\right)}
$$

Equação 1 - Percentuais de cristalização da lactose

Em que: $\mathrm{Cr}=$ Cristalização; $\mathrm{B}_{1}={ }^{\circ}$ Brix inicial (tempo zero); $\mathrm{B}_{2}={ }^{\circ}$ Brix final (após

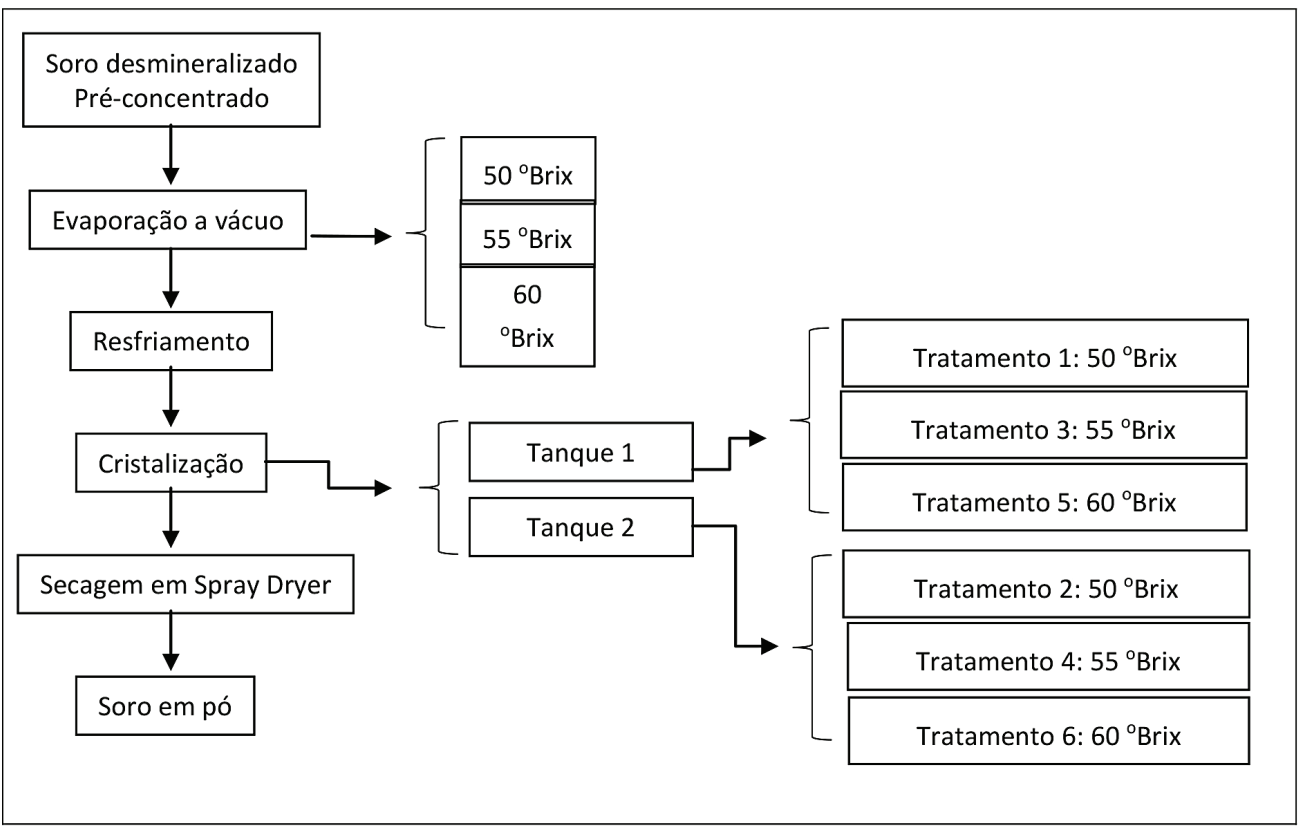

Figura 1 - Delineamento experimental aplicado no experimento 
cristalização); LAC $=\%$ de lactose no Extrato Seco Total; EST $=\%$ de Extrato Seco Total; as constantes 9500 e 95 referem-se ao fato que durante a cristalização da lactose o cristal formado possui $95 \%$ de sua massa molar proveniente da lactose $(342 \mathrm{~g} / \mathrm{mol})$ e $5 \%$ proveniente da agua de cristalização (18 g/mol).

Nesta etapa determinaram-se as equa- ções que relacionam o percentual de cristalização da lactose e sólidos solúveis com o tempo e a variação do teor de sólidos solúveis durante a cristalização através do software Excel $^{\circledR}$ 2014. Os dois pontos de amostragem utilizados no experimento estão representados na Figura 3, sendo as amostras coletadas simultaneamente em cada ponto em ambos os tanques.
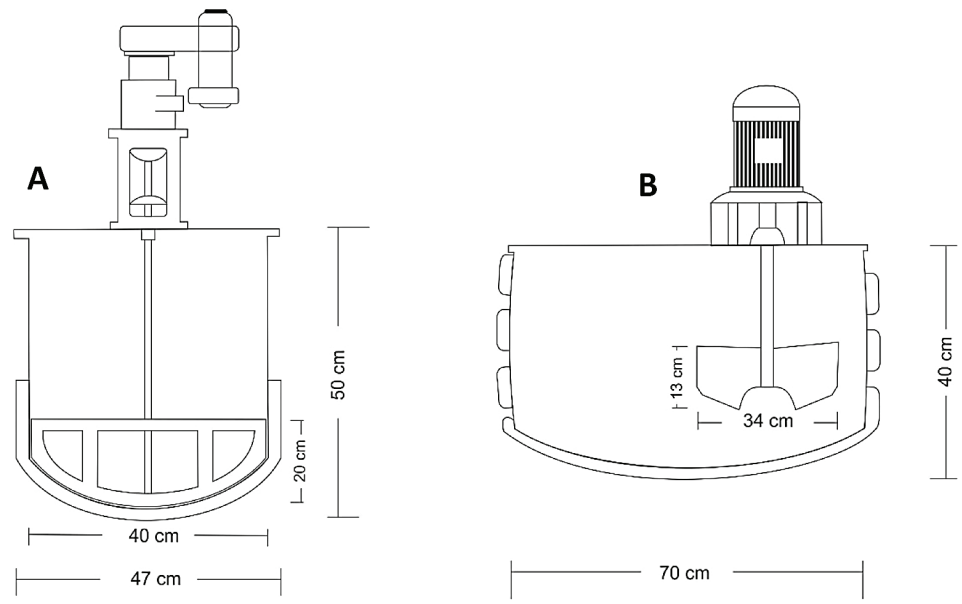

Figura 2 - A) Tanque de cristalização 1 - com agitador central; B) Tanque de cristalização 2 - com agitador periférico

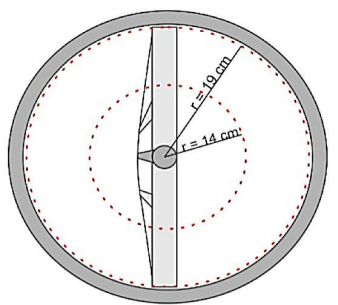

T1

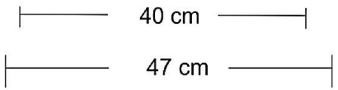

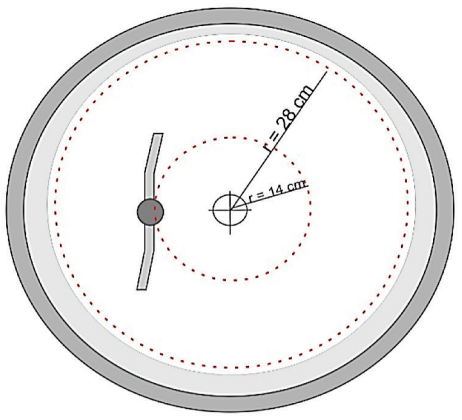

T2

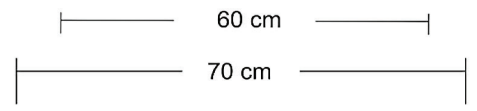

Figura 3 - Vista superior dos tanques de cristalização 1 (com agitador central) e 2 (com agitador lateral) e os respectivos raios para coleta de amostra 
Os dados obtidos das amostras coletadas com raio de $14 \mathrm{~cm}$ foram a amostragem padrão para todo experimento e os dados coletados das amostras na parede do equipamento (raio 19 e $28 \mathrm{~cm}$, respectivamente para o tanque 1 e 2), apenas para comparação de resultados, sendo eles apresentados na discussão deste trabalho.

Decorridos 240 minutos de cristalização o soro concentrado de cada um dos tanques foi transferido para cubas distintas de aço inoxidável e submetidos a refrigeração em câmara fria a $10{ }^{\circ} \mathrm{C}$, por aproximadamente 12 horas, sem agitação até a secagem. O soro refrigerado foi aquecido em banho maria até $40{ }^{\circ} \mathrm{C}$ e submetido a operação de secagem em um equipamento de 1 efeito da marca Niro Atomizer ${ }^{\circledR}$ modelo Minor Production com capacidade de secagem de $20 \mathrm{~kg} / \mathrm{h}$ e vazão de ar de secagem de $670 \mathrm{~kg} / \mathrm{h}$.

As condições técnicas de operação do spray dryer foram: $165,0 \pm 5,0{ }^{\circ} \mathrm{C}$ de temperatura no ar de entrada da câmara, 82,5 \pm $2,5{ }^{\circ} \mathrm{C}$ de temperatura no ar de saída. Os soros em pó obtidos foram embalados a vácuo e armazenados ao abrigo de luz.

As análises estatísticas foram obtidas por meio da análise de comparação de médias (anova) seguindo do teste de Tukey com nível de probabilidade de 0,05 . Para a comparação de medias de dados pareados foi utilizado o teste " $t$ " de Student $(\mathrm{p}=0,05)$.

As equações de ajuste das retas foram obtidas pelo Excel $^{\circledR}$ 2014. A determinação da composição físico-química se deu segundo Pereira (2001). O teor de proteínas foi determinado pelo método Kjeldahl, adotando o fator de conversão de nitrogênio para proteína de 6,38 (IDF, 1993); o conteúdo de lipídeos foi determinado pelo método de Gerber (FIL/ ISO, 2008); o teor de água total pelo método gravimétrico (IDF, 1988); o teor de resíduo mineral fixo (cinzas) seguindo o método gravimétrico após calcinação das amostras em mufla a $550{ }^{\circ} \mathrm{C}$ e o teor de lactose por diferença entre o teor de sólidos totais e os teores de sólidos determinados individualmente e somados. A atividade de água do soro em pó foi determinada a temperatura de $25{ }^{\circ} \mathrm{C}$, em um determinador Aqualab (Decagon 3TE, Decagon Devices Inc., USA).

A morfologia e a distribuição do tamanho das partículas das amostras de soro em pó foram avaliadas sem preparação prévia por microscopia eletrônica de varredura (Hitachi TM 3000, Hitachi Ltd., Tokyo, Japan). Três magnificações (50x, 300x e 2000x) foram utilizadas na caracterização das amostras. Além dos seis tratamentos, uma amostra de soro em pó comercial foi avaliada para efeitos de comparação. A distribuição do tamanho das partículas dos pós durante processo de reidratação foi obtida utilizando o analisador de difração a laser Beckman Coulter LS 13320 (Beckman Coulter, Miami, FL, EUA) acoplado ao módulo de análise liquida (Aqueous liquid module, Beckman Coulter, Miami, FL, EUA). Quantidades suficientes das amostras para gerar a turbidez necessária às leituras foram adicionadas ao reservatório do módulo de análise líquida contendo água a temperatura ambiente. A adição das amostras foi realizada lentamente para impedir a formação de aglomerados. O processo de reidratação dos pós durante recirculação no equipamento foi acompanhado durante $10 \mathrm{~min}$ através de coletas de dados de 1 min cada. Os resultados foram obtidos utilizando o índice de 1,332 para o meio dispersante (água) e 1,53 para as partículas (cristais de lactose) de acordo com Bushill et al. (1965); Mimouni et al. (2005) e foram representados pelo \% de volume ocupado pelas partículas em função do seu tamanho.

\section{RESULTADOS E DISCUSSÃO}

As condições de evaporação a vácuo empregadas para os três tratamentos (50 ${ }^{\circ}$ Brix, $55^{\circ}$ Brix e $60{ }^{\circ}$ Brix) seguido de seus valores médios e variações são apresentadas na Tabela 1 . 
A composição centesimal média dos soros concentrados desmineralizados obtido após concentração a pressões reduzidas obtido no experimento é apresentada na Tabela 2.
Na Tabela 3 encontra-se o teor de lactose nos diferentes soros concentrados em relação ao teor de umidade e de sólidos totais.

Tabela 1 - Atributos para a evaporação do soro pré-concentrado nos tratamentos realizados $\left(50{ }^{\circ}\right.$ Brix, $55^{\circ}$ Brix e $60^{\circ}$ Brix $)(n=3)$

\begin{tabular}{lccc}
\hline \multicolumn{1}{c}{ Atributos } & \multicolumn{3}{c}{ Valor } \\
\cline { 2 - 4 } & $50{ }^{\circ}$ Brix & $55^{\circ}$ Brix & $60{ }^{\circ}$ Brix \\
\hline $\begin{array}{l}\text { Temperatura de entrada do } \\
\text { soro pré-concentrado }\left({ }^{\circ} \mathrm{C}\right)\end{array}$ & $29,7 \pm 1,7$ & $23,2 \pm 2,0$ & $29,2 \pm 4,0$ \\
Temperatura de evaporação $\left({ }^{\circ} \mathrm{C}\right)$ & $65,8 \pm 6,0$ & $59,9 \pm 7,0$ & $57,4 \pm 6,7$ \\
$\begin{array}{l}\text { Temperatura da água condensada } \\
\text { na camisa }\left({ }^{\circ} \mathrm{C}\right)\end{array}$ & $65,2 \pm 8,4$ & $65,2 \pm 11,4$ & $72,0 \pm 11,3$ \\
$\begin{array}{l}\text { Pressão do vapor a alta pressão } \\
\text { (kgf/cm }{ }^{2} \text { ) }\end{array}$ & $1,7 \pm 0,7$ & $1,5 \pm 0,5$ & $1,2 \pm 0,4$ \\
$\begin{array}{l}\text { Pressão reduzida pelo sistema } \\
\text { de vácuo (cm Hg) }\end{array}$ & $55,7 \pm 3,1$ & $52,3 \pm 7,2$ & $53,5 \pm 5,4$ \\
$\begin{array}{l}\text { Teor de sólidos solúveis soro } \\
\text { pré-concentrado }\left({ }^{\circ} \text { Brix) }\right.\end{array}$ & $31,7 \pm 2,3$ & $33,9 \pm 0,4$ & $33,8 \pm 1,1$ \\
$\begin{array}{l}\text { Teor de sólidos final soro } \\
\text { concentrado }\left({ }^{\circ} \text { Brix) }\right.\end{array}$ & $49,3 \pm 1,3$ & $55,5 \pm 0,7$ & $60,5 \pm 1,8$ \\
Tempo em evaporação (minutos) & $188,3 \pm 57,7$ & $180,0 \pm 42,7$ & $161,6 \pm 72,3$ \\
Massa de soro (kg) & $102,8 \pm 10,5$ & $112,1 \pm 2,3$ & $106,8 \pm 6,4$ \\
$\begin{array}{l}\text { Fator de concentração } \\
\text { Massa de evaporado }(\mathrm{kg})\end{array}$ & $1,56 \pm 0,02$ & $1,75 \pm 0,15$ & $1,96 \pm 0,04$ \\
Taxa de evaporação $(\mathrm{kg} / \mathrm{h})$ & $36,9 \pm 4,5$ & $48,0 \pm 6,0$ & $52,3 \pm 2,2$ \\
\hline
\end{tabular}

Tabela 2 - Composição centesimal do soro concentrado desmineralizado obtido após concentração $(\mathrm{n}=3)$

\begin{tabular}{ccccccc}
\hline & $\begin{array}{c}\text { Umidade } \\
(\% \mathrm{~m} / \mathrm{m})\end{array}$ & $\begin{array}{c}\text { Sólidos } \\
\text { Totais } \\
(\% \mathrm{~m} / \mathrm{m})\end{array}$ & $\begin{array}{c}\text { Proteínas } \\
(\% \mathrm{~m} / \mathrm{m})\end{array}$ & $\begin{array}{c}\text { Gordura } \\
(\% \mathrm{~m} / \mathrm{m})\end{array}$ & $\begin{array}{c}\text { Lactose* } \\
(\% \mathrm{~m} / \mathrm{m})\end{array}$ & $\begin{array}{c}\text { RMF } \\
(\% \mathrm{~m} / \mathrm{m})\end{array}$ \\
\hline $50^{\circ}$ Brix & $51,5^{\mathrm{a}} \pm 1,4$ & $48,5^{\mathrm{c}} \pm 1,4$ & $5,3^{\mathrm{c}} \pm 0,3$ & $0,2^{\mathrm{a}} \pm 0,1$ & $38,7^{\mathrm{c}} \pm 1,4$ & $3,0^{\mathrm{a}} \pm 0,3$ \\
$55^{\circ}$ Brix & $45,4^{\mathrm{b}} \pm 1,4$ & $54,6^{\mathrm{b}} \pm 1,4$ & $6,8^{\mathrm{b}} \pm 0,7$ & $0,2^{\mathrm{a}} \pm 0,1$ & $46,0^{\mathrm{b}} \pm 1,3$ & $3,4^{\mathrm{a}} \pm 0,6$ \\
$60^{\circ}$ Brix & $36,9^{\mathrm{c}} \pm 1,4$ & $63,1^{\mathrm{a}} \pm 1,4$ & $8,1^{\mathrm{a}} \pm 0,5$ & $0,2^{\mathrm{a}} \pm 0,1$ & $51,7^{\mathrm{a}} \pm 1,2$ & $3,5^{\mathrm{a}} \pm 0,7$ \\
\hline
\end{tabular}

As médias seguidas da mesma letra na mesma coluna, não diferem entre si pelo Teste de Tukey, ao nível de $5 \%$ de probabilidade. * Calculado por diferença. RMF: resíduo mineral fixo. 
A taxa de resfriamento obtida desde a entrada do soro nos tanques de cristalização (tanque 1 e tanque 2) até o final da etapa de cristalização é apresentada pela Figura 4.

Nos primeiros 60 minutos de resfriamento atingiu-se a temperatura média de $31,7^{\circ} \mathrm{C} \pm 1,4^{\circ} \mathrm{C}$. Nesta fase do resfriamento a taxa média obtida foi de $-0,34{ }^{\circ} \mathrm{C} \pm 0,05{ }^{\circ} \mathrm{C} /$ min. Segundo o teste de Tukey (5\%) a taxa de resfriamento não apresentou diferença significativa entre as médias obtidas em todos os tratamentos e nos dois diferentes tanques de cristalização, confirmando a hipótese de que o resfriamento ocorreu nas mesmas condições em todo experimento.

Após os 60 minutos de cristalização a circulação de água na camisa dos tanques foi interrompida e o resfriamento ocorreu por meio da troca de calor com a água retida na camisa dos tanques de cristalização. A taxa de cristalização da lactose é apresentada na Figura 5.

Tabela 3 - Composição dos soros com diferentes concentrações obtidos por evaporação a vácuo $(\mathrm{n}=3)$

\begin{tabular}{cccc}
\hline Constituinte & $\begin{array}{c}\text { Soro } 50 \\
{ }^{\circ} \text { Brix }\end{array}$ & $\begin{array}{c}\text { Soro 55 } \\
{ }^{\circ} \text { Brix }\end{array}$ & $\begin{array}{c}\text { Soro 60 } \\
{ }^{\circ} \text { Brix }\end{array}$ \\
\hline Teor de sólidos totais $(\% \mathrm{~m} / \mathrm{m})$ & $48,5^{\mathrm{c}} \pm 1,4$ & $54,6^{\mathrm{b}} \pm 1,4$ & $63,1^{\mathrm{a}} \pm 1,4$ \\
PLST $(\% \mathrm{~m} / \mathrm{m})$ & $79,2^{\mathrm{b}} \pm 1,8$ & $83,6^{\mathrm{a}} \pm 1,2$ & $81,5^{\mathrm{ab}} \pm 1,2$ \\
MLAP $(\% \mathrm{~m} / \mathrm{m})$ & $75,7^{\mathrm{c}} \pm 4,3$ & $102,3^{\mathrm{b}} \pm 6,1$ & $140,7^{\mathrm{a}} \pm 6,6$ \\
\hline
\end{tabular}

Sendo: PLST $=$ porcentagem de lactose nos sólidos $(\% \mathrm{~m} / \mathrm{m})$, MLAP $=$ massa de lactose em $100 \mathrm{~g}$ de água do produto. As médias seguidas da mesma letra, não diferem entre si pelo Teste de Tukey, ao nível de 5\% de probabilidade.

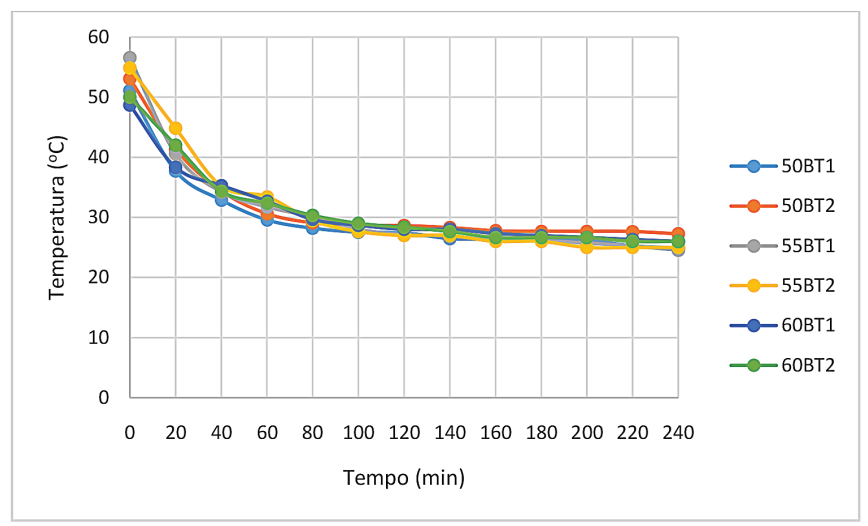

Figura 4 - Taxa de resfriamento do soro concentrado após evaporação à vácuo nos diferentes tratamentos analisados $(\mathrm{n}=3)$

Sendo: 50BT1: $50^{\circ}$ Brix no tanque de cristalização 1; 50BT2: $50^{\circ}$ Brix no tanque de cristalização 2; 55BT1: $55^{\circ}$ Brix no tanque de cristalização 1; 55BT2: $55^{\circ}$ Brix no tanque de cristalização 2 ; 60BT1: $60^{\circ}$ Brix no tanque de cristalização 1 e 60BT2: $60^{\circ}$ Brix no tanque de cristalização 2. 
A porcentagem de cristalização da lactose alcançada ao final dos 240 minutos foi diferente para cada tanque, sendo o tanque 1 (com agitador central), nas três concentrações estudadas, o que propiciou maiores valores para cristalização conforme a Tabela 4 .

Ao comparar as médias apresentadas na Tabela 4 referente aos tanques 1 e 2 em uma mesma concentração, aplicando o teste $\mathrm{t}$ de student (bi-caudal, $\alpha=0,05$ ), o valor de p encontrado foi de 0,$1771 ; 0,4038$ e 0,0272 respectivamente para as concentrações de $50{ }^{\circ}$ Brix, $55^{\circ}$ Brix e $60{ }^{\circ}$ Brix. Os resultados obtidos para ambas as concentrações rejeita a hipótese nula, ou seja, existe diferença entre as médias obtidas do tanque 1 em relação as médias do tanque 2 em todas as concentrações estudadas.

A diferença das médias dos percentuais de cristalização ( $\Delta \%$ Crist.) entre os tanques 1 e 2 foi decrescente a medida que foi aumentado o teor de sólidos solúveis do soro concentrado na saída do evaporador, tornando cada vez mais próximo os resultados obtidos nos dois tanques com o aumento da concentração ao final da evaporação, sendo uma relação não linear.

Observou-se por meios não analíticos que dentre os tratamentos estudados, aqueles com teores mais elevados de sólidos solúveis

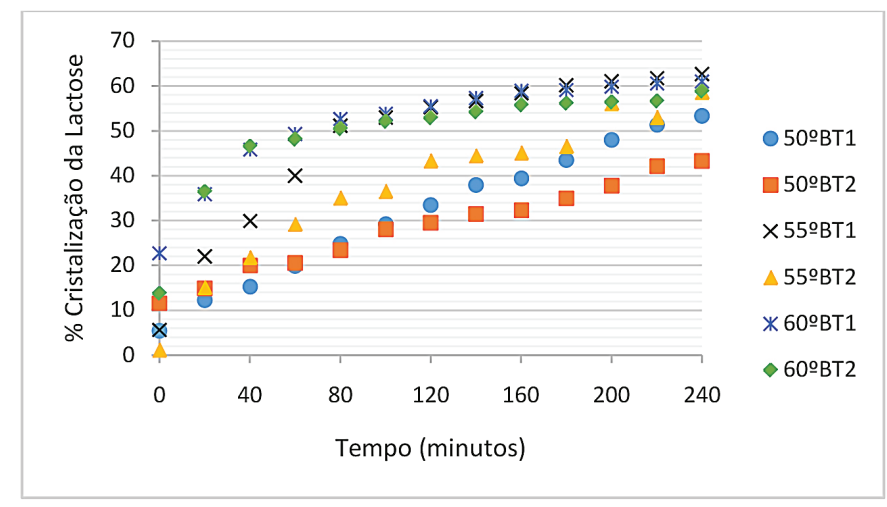

Figura 5 - Comportamento cinético de formação de cristais de lactose em soro concentrado $\left(50{ }^{\circ}\right.$ Brix, $55^{\circ}$ Brix e $60^{\circ}$ Brix $)$, durante etapa de cristalização $(\mathrm{n}=3)$

Sendo: T1: tanque de cristalização 1 e T2: tanque de cristalização 2.

Tabela 4 - Percentual de cristalização da lactose alcançado ao término da etapa de cristalização $(\mathrm{n}=3)$

\begin{tabular}{cccc}
\hline & \multicolumn{3}{c}{ Percentual de cristalização da lactose (\%) } \\
\hline & $50^{\circ}$ Brix & $55^{\circ}$ Brix & $60{ }^{\circ}$ Brix \\
\hline Tanque 1 & $53,3 \pm 2,3$ & $62,6 \pm 8,6$ & $61,1 \pm 8,4$ \\
Tanque 2 & $43,3 \pm 7,7$ & $58,6 \pm 6,2$ & $58,9 \pm 7,8$ \\
$\Delta$ \%Crist. & $10,0 \pm 5,0$ & $4,0 \pm 7,4$ & $2,2 \pm 8,1$ \\
\hline
\end{tabular}

Sendo: $\Delta \%$ Crist. $=$ a variação no percentual de cristalização entre os tanques. 
no soro apresentaram um aumento de sua viscosidade aparente ("viscosidade"), desta forma, uma maior resistência à agitação é gerada em ambos os tanques de cristalização, fazendo com que o fenômeno físico de agitação não ocorra de forma eficiente para os diferentes tratamentos, visto que o processo ocorreu em condições padronizadas.

Ao elevar o teor de sólidos solúveis inicial para $60^{\circ}$ Brix os efeitos cinéticos e de "viscosidade" começam a perder força frente à força motriz de saturação, apresentando taxa de cristalização próxima ao do tratamento com $55{ }^{\circ}$ Brix. De acordo com estes resultados, principalmente a $50{ }^{\circ} \mathrm{Brix}$ há diferença entre os tipos de cristalizadores no tocante a cristalização da lactose em relação a porcentagem de lactose cristalizada obtida, indicando que o efeito cinético (modo de agitador) é mais pronunciado do que o efeito termodinâmico do estado de equilíbrio (saturação de lactose) que é a força motriz para cristalização.

Para otimizar o processo visto a perda do efeito cinético frente ao aumento da "vis- cosidade", uma maior velocidade de agitação do soro é necessária durante a etapa de cristalização, calculada de acordo com a concentração que se deseja trabalhar, podendo assim, obter o maior aproveitamento dos efeitos cinético e termodinâmico do estado de equilíbrio, favorecido pela agitação e saturação da lactose respectivamente.

Os percentuais de cristalização da lactose encontrados para todos tratamentos apresentados (Tabela 4) não estão de acordo com os descritos na literatura para processamento de soro desnatado com cristalização após a evaporação a vácuo (SCHUCK et al. 2004; WESTERGAARD, 2001; KNIPSCHILDT; ANDERSEN, 1994). Segundo Písecký (1997), um soro concentrado de boa qualidade para secagem deve apresentar um percentual de cristalização da lactose de no mínimo 70\%, o que não foi possível ser obtido nas condições do experimento. Atualmente recomenda-se a concentração soro entre $50{ }^{\circ}$ Brix e $60{ }^{\circ}$ Brix, visando a cristalização da lactose para a produção de soro em pó (SCHUCK et al., 2012).

Tabela 5 - Equações matemáticas resultantes do estudo da cristalização do soro concentrado ( 50 B Brix $)$ em dois modelos diferentes de cristalizadores

\begin{tabular}{|c|c|c|c|}
\hline Tratamento & Tipo de relação & Equação & $\begin{array}{c}\text { Equação e coeficiente } \\
\text { de correlação }\end{array}$ \\
\hline $50^{\circ}$ Brix T1 & & I & $y=-0,0469 x+49,895$ \\
\hline & $\begin{array}{c}\text { Relação entre tempo (minutos) (x) } \\
\mathrm{e}^{\circ} \mathrm{Brix}(\mathrm{y})\end{array}$ & & $\mathrm{R}^{2}=0,9975$ \\
\hline $50^{\circ}$ Brix T2 & & II & $\begin{array}{c}y=-0,0294 x+48,716 \\
R^{2}=0,9877\end{array}$ \\
\hline $50^{\circ}$ Brix T1 & & III & $y=0,1576 x+6,3496$ \\
\hline & $\begin{array}{l}\text { Relação entre tempo (minutos) }(x) \\
\text { e \% de cristalização da lactose }(y)\end{array}$ & & $\mathrm{R}^{2}=0,9922$ \\
\hline $50^{\circ}$ Brix T2 & & IV & $\begin{array}{c}\mathrm{y}=0,1268 \mathrm{x}+13,23 \\
\mathrm{R}^{2}=0,986\end{array}$ \\
\hline
\end{tabular}

Sendo: T1: Tanque de cristalização 1 (agitador central) e T2: Tanque de Cristalização 2 (agitador periférico). 
Tabela 6 - Equações matemáticas resultantes do estudo da cristalização do soro concentrado ( $55^{\circ}$ Brix) em dois modelos diferentes de cristalizadores

\begin{tabular}{|c|c|c|c|}
\hline Tratamento & Tipo de relação & Equação & $\begin{array}{c}\text { Equação e coeficiente } \\
\text { de correlação }\end{array}$ \\
\hline $55^{\circ}$ Brix T1 & & I & $y=-0,06 x+51,233$ \\
\hline & $\begin{array}{l}\text { Relação entre tempo (minutos) (x) } \\
\mathrm{e}^{\mathrm{o}} \mathrm{Brix}(\mathrm{y})\end{array}$ & & $\mathrm{R}^{2}=0,8406$ \\
\hline $55^{\circ}$ Brix T2 & & II & $\begin{array}{c}y=-0,0546 x+54,03 \\
R^{2}=0,9477\end{array}$ \\
\hline $55^{\circ}$ Brix T1 & & III & $y=0,1927 x+21,681$ \\
\hline & $\begin{array}{l}\text { Relação entre tempo (minutos) (x) } \\
\text { e \% de cristalização da lactose (y) }\end{array}$ & & $\mathrm{R}^{2}=0,7903$ \\
\hline $55^{\circ}$ Brix T2 & & IV & $\begin{array}{c}\mathrm{y}=0,1909 x+11,484 \\
\mathrm{R}^{2}=0,9169\end{array}$ \\
\hline
\end{tabular}

Sendo: T1: Tanque de cristalização 1 (agitador central) e T2: Tanque de Cristalização 2 (agitador periférico).

Tabela 7 - Equações matemáticas resultantes do estudo da cristalização do soro concentrado $\left(60{ }^{\circ}\right.$ Brix $)$ em dois modelos diferentes de cristalizadoress

\begin{tabular}{|c|c|c|c|}
\hline Tratamento & Tipo de relação & Equação & $\begin{array}{c}\text { Equação e coeficiente } \\
\text { de correlação }\end{array}$ \\
\hline $60^{\circ}$ Brix T1 & & I & $y=0,1426 x+34,934$ \\
\hline & $\begin{array}{c}\text { Relação entre tempo (minutos) (x) } \\
\mathrm{e}^{\circ} \operatorname{Brix}(\mathrm{y})\end{array}$ & & $\mathrm{R}^{2}=0,7462$ \\
\hline $60^{\circ}$ Brix T2 & & II & $\begin{array}{c}\mathrm{y}=0,1413 \mathrm{x}+31,939 \\
\mathrm{R}^{2}=0,6156\end{array}$ \\
\hline $60^{\circ}$ Brix T1 & & III & $y=-0,0424 x+52,859$ \\
\hline & $\begin{array}{l}\text { Relação entre tempo (minutos) }(x) \\
\text { e \% de cristalização da lactose }(y)\end{array}$ & & $\mathrm{R}^{2}=0,8119$ \\
\hline $60^{\circ}$ Brix T2 & & IV & $\begin{array}{c}y=-0,0396 x+53,584 \\
R^{2}=0,6959\end{array}$ \\
\hline
\end{tabular}

Sendo: T1: Tanque de cristalização 1 (agitador central) e T2: Tanque de Cristalização 2 (agitador periférico). 
Com o objetivo de atingir o teor mínimo de $70 \%$ de cristalização, três ações seriam necessárias: um maior tempo de permanência no cristalizador (tempo superior a 240 minutos); maior velocidade de agitação; e/ou presença de uma maior quantidade de núcleos de cristalização. A partir das curvas da cinética de cristalização obtidas determinaram-se as equações de ajuste para todos os tratamentos estudados, sendo apresentadas diferentes relações entre seus termos (Tabelas 5, 6 e 7).

A utilização da equação I e II permite determinar o tempo $(\mathrm{x})$ para se atingir o ${ }^{\circ}$ Brix (y) desejado, o que possui importante aplicação industrial, visto que a medição do teor de sólidos solúveis em ${ }^{\circ}$ Brix é a forma amplamente utilizada para controle da cristalização da lactose em soro de leite concentrado.

Ao avaliarmos as Tabelas 5, 6 e 7 é possivel discutir o efeito do aumento do teor de lactose na solução ( ${ }^{\circ}$ Brix mais elevado) com os coeficientes de ajuste ao modelo linear $\left(\mathrm{R}^{2}\right)$. Quando a concentração da lactose é menor na solução, os efeitos da agitação são mais pronunciados, desta forma, observa-se para o tratamento com $50{ }^{\circ}$ Brix que as equações matemáticas apresentam valores mais elevados para $\mathrm{R}^{2}$.

A medida que o teor de lactose aumenta na solução (atingindo a zona de supersaturação) a agitação possui menor efeito sobre a cristalização, devido a nucleação expontanea ser intensa como consequência da maior força motriz a cristalização, fazendo com que os valores para $\mathrm{R}^{2}$ fossem mais baixos (tratamentos $55^{\circ} \mathrm{Brix}$ e $60^{\circ} \mathrm{Brix}$ ).

As equações III e IV apresentadas (Tabela 7) podem ser empregadas para prever o tempo necessário para um percentual de cristalização desejado em condições controladas de cristalização em soro desnatado desmineralizado, apresentando grande valia prática, pois possibilitam a previsão e controle do processamento industrial, conforme os atributos estabelecidos. Ao aplicar as equações III e IV foi encontrado o tempo mínimo em minutos desde a saída do evaporador para que $70 \%$ da lactose fosse cristalizada (Tabela 8).

Tabela 8 - Tempo em minutos necessário para atingir no mínimo $70 \%$ de cristalização da lactose em soro concentrado para cada tratamento

\begin{tabular}{cc}
\hline Tratamento & $\begin{array}{c}\text { Tempo (minutos) } \\
\text { para atingir 70\% de } \\
\text { cristalização da lactose }\end{array}$ \\
\hline $50^{\circ}$ Brix T1 & 404 \\
$50^{\circ}$ Brix T2 & 448 \\
$55^{\circ}$ Brix T1 & 250 \\
$55^{\circ}$ Brix T2 & 306 \\
$60^{\circ}$ Brix T1 & 404 \\
$60^{\circ}$ Brix T2 & 414 \\
\hline
\end{tabular}

Sendo: T: tanque.

Conforme a Tabela 8 o tanque de cristalização 2 (com agitador periférico) apresentou um maior tempo necessário para alcançar o mínimo de $70 \%$ de cristalização da lactose desejável, indicando uma menor eficiência cinética quanto ao tipo de agitação em relação ao tanque 1 (com agitador central) que em todas concentrações avaliadas apresentaram menor tempo. Durante o experimento foram coletadas amostras simultaneamente do centro e próximo a parede do tanque, conforme ilustrado na Figura 3.

Observando os dados apresentados na Tabela 9 na concentração de $50{ }^{\circ}$ Brix não houve diferença entre as regiões central e próxima a parede indicando uma agitação homogênea em toda área do tanque. O tratamento com $55{ }^{\circ}$ Brix apresentou uma maior porcentagem de cristalização na região central em ambos os tanques, configurando uma cristalização não homogênea, indicando necessitar de um maior efeito cinético de agitação nos tanques. $\mathrm{O}$ tratamento com $60^{\circ} \mathrm{Brix}$ 
apresentou cristalização nas duas regiões idênticas, apesar de ter a contribuição cinética comprometida pela maior "viscosidade", o efeito termodinâmico do estado de equilíbrio foi maior devido a saturação da lactose nesta concentração, obtendo, portanto uma cristalização homogênea em toda região dos dois tanques.

Os resultados das análises físico-químicas dos pós são apresentados na Tabela 10 .

De acordo com a Tabela 10 todos os soros em pó apresentaram composição físicoquímica sem diferença significativa entre as médias segundo teste de Tukey, ao nível de $5 \%$ de probabilidade, devido a padronização das condições de operação do equipamento no processo de secagem.

A atividade de água e o teor de umidade do pó são apresentados na Tabela 11 .

As atividades de água apresentaram valores inferiores ao recomendado para produtos lácteos desidratados, próxima a 0,20, sendo esse valor o mais adequado para o controle das reações químicas de degradação nestes produtos (SCHUCK et al., 2008). O valor abaixo de 0,20 para o soro em pó desnatado é um valor aceitável, uma vez que os constituintes que mais contribuem para as reações químicas de oxidação são os lipídeos.

Tabela 9 - Comparação entre o teor de sólidos solúveis e \% de cristalização da lactose nas diferentes concentrações analisadas avaliando regiões diferentes de amostragem em cada um dos tanques $(\mathrm{n}=3)$

\begin{tabular}{|c|c|c|c|c|c|c|c|c|c|c|c|c|}
\hline & \multicolumn{6}{|c|}{ Tanque 1} & \multicolumn{6}{|c|}{ Tanque 2} \\
\hline & \multicolumn{3}{|c|}{ Parede } & \multicolumn{3}{|c|}{ Centro } & \multicolumn{3}{|c|}{ Parede } & \multicolumn{3}{|c|}{ Centro } \\
\hline & B1 & B2 & $\% \mathrm{Cr}$ & B1 & B2 & $\% \mathrm{Cr}$ & B1 & B2 & $\% \mathrm{Cr}$ & B1 & B2 & $\% \mathrm{Cr}$ \\
\hline $50 \mathrm{~B}$ & 50 & 39 & 53 & 50 & 39 & 53 & 49 & 41 & 44 & 49 & 42 & 43 \\
\hline $55 \mathrm{~B}$ & 54 & 41 & 55 & 56 & 39 & 63 & 55 & 44 & 47 & 56 & 41 & 59 \\
\hline $60 \mathrm{~B}$ & 59 & 44 & 61 & 57 & 44 & 61 & 60 & 45 & 59 & 59 & 45 & 59 \\
\hline
\end{tabular}

Sendo: B: Brix; B1: Brix inicial; B2: Brix final e Cr: Cristalização.

Tabela 10 - Composição físico-química do soro em pó obtido $(\mathrm{n}=3)$

\begin{tabular}{ccccccc}
\hline Trat. & $\begin{array}{c}\text { Umidade } \\
(\% \mathrm{~m} / \mathrm{m})\end{array}$ & $\begin{array}{c}\text { Sólidos Totais } \\
(\% \mathrm{~m} / \mathrm{m})\end{array}$ & $\begin{array}{c}\text { Proteínas } \\
(\% \mathrm{~m} / \mathrm{m})\end{array}$ & $\begin{array}{c}\text { Gordura } \\
(\% \mathrm{~m} / \mathrm{m})\end{array}$ & $\begin{array}{c}\text { Lactose } \\
(\% \mathrm{~m} / \mathrm{m})\end{array}$ & $\begin{array}{c}\text { RMF } \\
(\% \mathrm{~m} / \mathrm{m})\end{array}$ \\
\hline 50BT1 & $4,4^{\mathrm{a}} \pm 0,4$ & $95,7^{\mathrm{a}} \pm 0,4$ & $12,9^{\mathrm{a}} \pm 1,6$ & $0,6^{\mathrm{a}} \pm 0,3$ & $76,7^{\mathrm{a}} \pm 2,1$ & $5,3^{\mathrm{a}} \pm 0,6$ \\
50BT2 & $4,1^{\mathrm{a}} \pm 0,6$ & $95,9^{\mathrm{a}} \pm 0,7$ & $12,6^{\mathrm{a}} \pm 0,8$ & $0,7^{\mathrm{a}} \pm 0,2$ & $76,9^{\mathrm{a}} \pm 1,1$ & $5,8^{\mathrm{a}} \pm 0,5$ \\
55BT1 & $4,2^{\mathrm{a}} \pm 1,5$ & $95,5^{\mathrm{a}} \pm 1,5$ & $10,0^{\mathrm{a}} \pm 3,2$ & $0,4^{\mathrm{a}} \pm 0,2$ & $79,2^{\mathrm{a}} \pm 3,8$ & $5,9^{\mathrm{a}} \pm 1,2$ \\
55BT2 & $4,8^{\mathrm{a}} \pm 1,7$ & $95,2^{\mathrm{a}} \pm 1,7$ & $9,9^{\mathrm{a}} \pm 4,7$ & $0,4^{\mathrm{a}} \pm 0,2$ & $79,0^{\mathrm{a}} \pm 4,6$ & $5,6^{\mathrm{a}} \pm 0,1$ \\
60BT1 & $4,3^{\mathrm{a}} \pm 0,4$ & $96,4^{\mathrm{a}} \pm 1,8$ & $12,9^{\mathrm{a}} \pm 1,4$ & $0,7^{\mathrm{a}} \pm 0,3$ & $76,3^{\mathrm{a}} \pm 1,8$ & $5,5^{\mathrm{a}} \pm 0,3$ \\
60BT2 & $4,1^{\mathrm{a}} \pm 0,6$ & $95,9^{\mathrm{a}} \pm 0,6$ & $12,2^{\mathrm{a}} \pm 0,9$ & $0,7^{\mathrm{a}} \pm 0,3$ & $77,2^{\mathrm{a}} \pm 0,9$ & $5,8^{\mathrm{a}} \pm 0,5$ \\
\hline
\end{tabular}

Sendo: Trat.: Tratamento; B: Brix; T: tanque de cristalização e RMF: resíduo mineral fixo. As médias seguidas da mesma letra na mesma coluna, não diferem entre si pelo Teste de Tukey, ao nível de 5\% de probabilidade.

* Calculado por diferença. 
O soro quando cristalizado no tanque 2 (com agitador central), após secagem em spray dryer em condições controladas, apresentou maior deposição de pó na superfície do equipamento, exceto para o tratamento de $60{ }^{\circ}$ Brix, onde a diferença foi minimizada devido a uma cristalização mais homogênea entre os tanques. O fenômeno de adesão ou aderência ocorre com frequência em alimentos ricos em açúcares de baixa massa molar, que, no estado vítreo, são geralmente muito higroscópicos e apresentam temperaturas de transição vítrea $\left(\mathrm{T}_{\mathrm{g}}\right.$ ) baixa (BRENNAN, 1994). Sendo assim, os tratamentos que utilizaram o tanque 2 e apresentaram uma maior diferença entre a \% de cristalização obtida foram os que mais aderiram nas paredes dos equipamentos, possivelmente por apresentarem uma menor $\left(\mathrm{T}_{\mathrm{g}}\right)$. Segundo Chen; Patel (2008); Verdurmen et al. (2006) quanto menor for a $\mathrm{T}_{\mathrm{g}}$, mais estará sujeito a sofrer $\mathrm{o}$ fenômeno de stickiness, que está diretamente associado à tendência de alguns materiais à aglomeração ou adesão quando em contato com uma superfície durante e após a secagem.

Na Figura 6 seguem imagens da microscopia eletrônica das amostras de soro em pó obtidas pelo experimento com aumento de 2000x.

Ao observar a morfologia dos pós (Figura 6) é possível identificar a presença de poros na superfície das partículas, o que ocorreu em todas as amostras analisadas neste experimento. Durante a secagem por atomização no equipamento piloto empregado neste experimento a evaporação da água ocorreu em um único estágio, com um tempo de residência curto quando comparado com equipamentos industriais que possuem múltiplos estágios, portando, são necessárias maiores temperaturas do ar de saída para que a evaporação ocorra até o teor final de umidade desejado no pó (PÍSECKÝ, 1997; SCHUCK, 2009). A presença destes poros foi diferente entre os tratamentos analisados, sendo maior quando utilizado soro com menor teor de sólidos solúveis na secagem. Essa diferença pode ser devido a alguns fatores como: "viscosidade" mais baixa do soro concentrado, maior quantidade de água a ser evaporada no produto com $50{ }^{\circ} \mathrm{Brix}$ em comparação com os demais; e maior teor de lactose amorfa (mais água ligada).

Desta forma, neste experimento observa-se que mudanças nas condições de cristalização do soro concentrado (diferentes tanques de cristalização) e no teor de sólidos solúveis implicam na obtenção de pós com diferentes propriedades de reidratação, de adesão e com teores distintos de lactose cristalizada, impactando na conservação durante o prazo de validade.

Tabela 11 - Teores de umidade e de atividade de água médios para os soro em pó obtidos em concentrações diferentes nos tanques de cristalização 1 e $2(n=3)$

\begin{tabular}{lccc}
\hline & Atividade de Água $\left(\mathrm{A}_{\mathrm{w}}\right)$ & Teor de Água $(\% \mathrm{~m} / \mathrm{m})$ & Correlação de Pearson \\
\hline $50^{\circ}$ Brix T1 & $0,17^{\mathrm{a}} \pm 0,04$ & $4,4^{\mathrm{a}} \pm 0,4$ & 0,90 \\
$50^{\circ}$ Brix T2 & $0,15^{\mathrm{a}} \pm 0,02$ & $4,1^{\mathrm{a}} \pm 0,6$ & 0,77 \\
$55^{\circ}$ Brix T1 & $0,15^{\mathrm{a}} \pm 0,04$ & $4,2^{\mathrm{a}} \pm 1,5$ & $-0,35$ \\
$55^{\circ}$ Brix T2 & $0,19^{\mathrm{a}} \pm 0,08$ & $4,8^{\mathrm{a}} \pm 1,7$ & 0,93 \\
$60^{\circ}$ Brix T1 & $0,19^{\mathrm{a}} \pm 0,03$ & $4,3^{\mathrm{a}} \pm 0,4$ & $-0,21$ \\
$60^{\circ}$ Brix T2 & $0,18^{\mathrm{a}} \pm 0,04$ & $4,1^{\mathrm{a}} \pm 0,6$ & 0,23 \\
\hline
\end{tabular}

Sendo: $\mathrm{T}$ = tanque de cristalização. As médias seguidas da mesma letra na mesma coluna, não diferem entre si pelo Teste de Tukey, ao nível de $5 \%$ de probabilidade. * Calculado por diferença. 


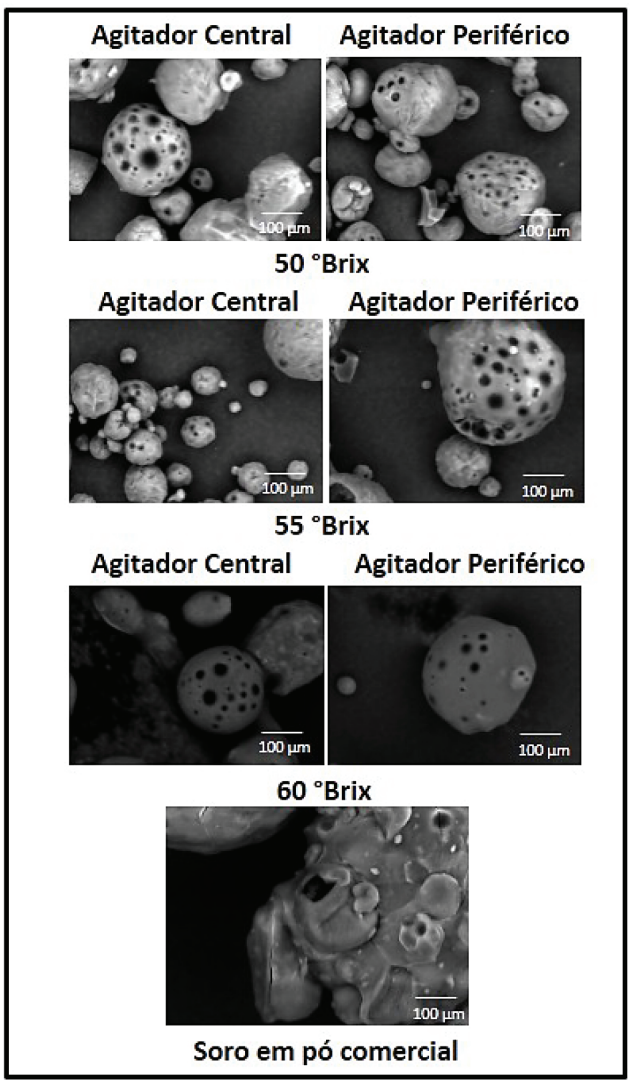

Figura 6 - Microscopia eletrônica de varredura de cada tratamento avaliado e de um soro em pó comercial (aumento 2000x)

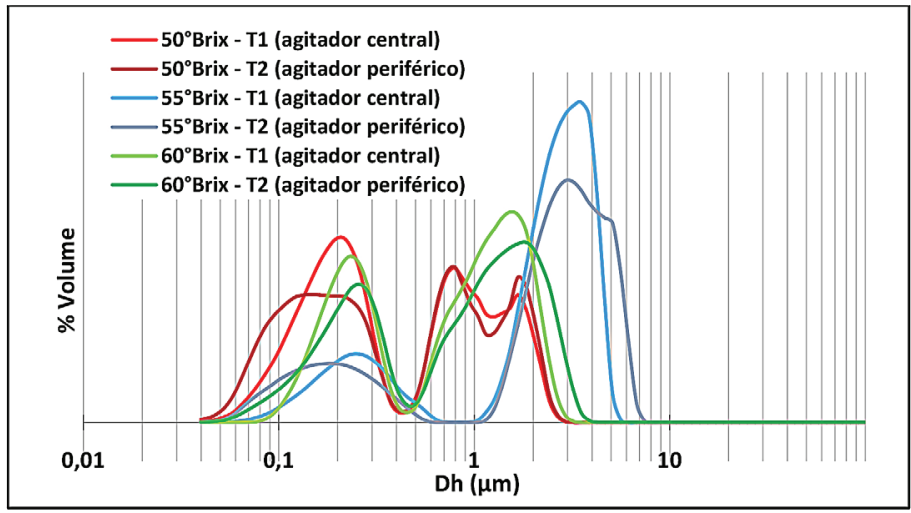

Figura 7 - Comparação da distribuição dos tamanhos das partículas das amostras durante a reidratação por recirculação no equipamento de difração a laser 
A evolução da distribuição do tamanho das partículas durante o acompanhamento da reidratação dos soros em pó em água foi monitorada mediante recirculação durante 10 minutos no equipamento de difração a laser. Independentemente dos tratamentos, houve pouca variação na distribuição das partículas entre 1 e 10 minutos de reidratação por recirculação no equipamento. A Figura 7 apresenta as curvas de reidratação obtidas após 5 minutos de recirculação e mostram a distribuição do tamanho das partículas (diâmetro hidrodinâmico, Dh - escala logarítmica) pela porcentagem do volume ocupado por elas.

As curvas apresentadas foram obtidas após 5 minutos de recirculação e mostram a distribuição do tamanho das partículas (diâmetro hidrodinâmico, Dh - escala logarítmica) pela porcentagem do volume ocupado por elas. $\mathrm{Na}$ concentração de $50{ }^{\circ} \mathrm{Brix}$ foram observados 3 populações, uma de menor tamanho $(0,05-0,4 \mu \mathrm{m})$ e as outras duas de maior tamanho $(0,5-2,5 \mu \mathrm{m})$ e partículas com tamanho máximo de aproximadamente 2,5 $\mu \mathrm{m}$. Nesta concentração a reidratação ocorreu de forma mais rápida que os demais tratamentos, devido à menor porcentagem de cristalização obtida comparado as outras concentrações e consequentemente uma maior quantidade de lactose amorfa presente, o que facilita o processo de reidratação.

Na concentração de $55^{\circ}$ Brix houve a presença de 2 populações, sendo a menor entre 0,05 a $0,60 \mu \mathrm{m}$ e a maior população com partículas de 1 a $8 \mu \mathrm{m}$. A segunda população observada foi dominante representando um maior volume. Neste tratamento ocorreu uma cristalização mais efetiva, apresentando uma maior quantidade de cristais de lactose no soro concentrado, demandando um maior tempo para solubilizar os cristais presentes, dificultando o processo de reidratação. $\mathrm{O}$ tanque 1 se diferenciou do 2 alcançando uma maior população, devido a diferença entre a taxa de cristalização obtida em cada um dos tanques.

O tratamento de $60{ }^{\circ}$ Brix também apresentou 2 populações $(0,1-0,5 \mu \mathrm{m}$ e 0,5 $-4 \mu \mathrm{m}$ ) praticamente equivalentes em volume e com partículas de tamanho máximo com aproximadamente $2,5 \mu \mathrm{m}$. Este tratamento foi o que apresentou resultados mais próximos entre o T1 e T2, devido principalmente a menor diferença entre a taxa obtida na etapa de cristalização nestes dois tanques.

\section{CONCLUSÕES}

Com este trabalho é possível concluir que o efeito cinético propiciado pelo tipo de agitação em diferentes tanques de cristalização (T1 - agitador central e T2 - agitador lateral), foram significativos na taxa cristalização do lactose em soro de leite concentrado obtida, mantendo as demais condições de cristalização constantes (taxa de resfriamento, temperatura, tempo e concentração), sendo o tanque com agitador central o que gerou maior quantidade de lactose na forma cristalina em todas concentrações avaliadas (50, 55 e $60{ }^{\circ}$ Brix). Em relação aos produtos obtidos, neste experimento observa-se que mudanças nas condições de cristalização do soro concentrado (em diferentes tanques) e no teor de sólidos solúveis implicam na obtenção de pós com diferentes propriedades de reidratação, de adesão e com teores distintos de lactose cristalizada, impactando na conservação durante o prazo de validade.

\section{AGRADECIMENTOS}

Os autores agradecem ao CNPq, CAPES e FAPEMIG pelo apoio financeiro concedido para realização do projeto e pelas bolsas de produtividade e de pós-doutorado dos autores AFC e ITP. Agradecem também ao Laticinios Porto Alegre pelo apoio e parceria na realização do experimento, em especial a toda equipe técnica. 


\section{REFERÊNCIAS}

BRENNAN, J. G. Food Dehydration: A Dictionary and Guide. [S.1.]: Taylor \& Francis, 1994.

BUSHILL J. H. et al. The crystallisation of lactose with particular reference to its occurrence in milk powder. Journal of Science Food Agriculture, v. 16, p. 622628, 1965.

CHEN, X. D.; PATEL, K. C. Manufacturing Better Quality Food Powders from Spray Drying and Subsequent Treatments. Drying Technology, v. 26, n. 11, p. 1313-1318, 2008.

INTERNATIONAL DAIRY FEDERATION (IDF). Determination of milk proteins. Brussels, 20B: 1993.

INTERNATIONAL DAIRY FEDERATION (IDF). Determination of gravimetric, drying method. Brussels, 15B:1988.

ISO. International Organization for Standardization, 2008b. International Standard. ISO 1737:2008(E)/IDF 13:2008(E). Evaporated Milk and Sweetened Condensed Milk - Determination of Fat Content Gravimetric Method (Reference method). Geneva, Switzerland.

KNIPSCHILDT, M. E.; ANDERSEN, G. G. In: ROBINSON, R. K. Advances in Milk Processing, London: Chapmam \& Hall, 1994. v. 1, p. 159-254.

MASTERS, K. Spray Drying in Practices. Copenhagen: Spraydry Consult International ApS, 2002. 464 p.

MIMOUNI, A. et al. Kinetics of lactose crystallization and crystal size as monitored by refractometry and laser light scattering: effect of proteins. Le Lait, v. 85, n. 4-5, p. 253-260, 2005.
PÍSECKÝ, I. J. Handbook of milk powder manufacture. Copenhagen: NIRO A/S, p. 261, 1997.

PEREIRA, D. B. C. et al. Físico-química do leite e derivados - Métodos Analíticos. $2^{\mathrm{a}} \mathrm{ed}$. Juiz de Fora: Oficina de Impressão Gráfica e Editora, 2001. 190p.

SCHUCK, P. Understanding the factors affecting spray-dried dairy powder properties and behaviour. In: CORREDIG, M. Dairy-derived ingredients - Food and nutraceutical uses. Woodhead Publishing, 2009. p. 24-50.

SCHUCK, P. et al. Séchage dês lactosérums et derives: role du lactose et de ladynamique de 1'eau. Le Lait, Rennes, v. 84, p. 243-268, 2004.

SCHUCK, P. et al. Analytical methods for food and dairy powders. Chichester: WileyBlackwell, 2012, p. 1-41.

SHUCK, P. et al. Relative humidity of outlet air: the key parameter to optimize moisture content and water activity of dairy powders. Dairy Science and Technology, v. 88, p. 45-52, 2008.

VERDURMEN, R. E. M. et al. Agglomeration in Spray Drying Installations (The EDECAD Project): Stickiness Measurements and Simulation Results. Drying Technology, v. 24, n. 6, p. 721-726, 2006.

WESTERGAARD, V. Tecnologia de La Leche en Polvo - Evaporación y Secado por Atomización. Copenhagen: Niro A/S, 2001. $166 \mathrm{p}$.

WESTERGAARD, V. Milk powder technology. In: Evaporation and spray drying. Copenhagen: Niro A/S, 2004. 168p. 